\title{
Focal vs. global ways of motion event processing and the role of language: Evidence from categorization tasks and eye tracking
}

\author{
Efstathia Soroli \\ Department of Linguistics, University of Lille, France \\ https://doi.org/10.36505/ExLing-2018/09/0026/000359
}

\begin{abstract}
Past research has shown that cultural experience and language specificities affect how people process spatial information cognitively. Holistic cognition is generally associated with East-Asian cultures while analytic processing to the Western ones. Similarly, the languages of the world vary greatly: some (verb-framed) invite to encode mainly general, core spatial components (Path) avoiding encodings related to the process of motion (Manner), while others (satellite-framed) focus on Manner systematically adding information about Path. The current study asked whether speakers who share the same cultural background (Western) but speak different languages (English (satellite-framed), French (verb-framed) and Greek (parallel)) show differences in categorization and visual processing of motion events. The findings show that holistic processing is not exclusive to Eastern cultures: speakers of indo-european are also influenced by the degree of focality of their language.

Key words: holistic vs. analytic cognition, focal vs. global strategies, spatial language, categorization, eye movements.
\end{abstract}

\section{Introduction}

There is a widespread idea that the human cognitive system functions the same, at least for all normal humans. This assumption of universality was recently strengthened by genetic theories that support the idea of a common genetic basis of the cognitive system (e.g., Kovas \& Plomin, 2006) and by the Minimalist Program (Chomsky, 2014) according to which humans are all equipped with the same set of general conceptual categories that allows for processing of core features irrespective of linguistic or cultural background.

A growing number of studies, however, show that experience and more specifically experience with language may be one of the formative or even transformative aspects of human cognition. Work by Nisbett et al. (2001) and Soroli et al. (2018) reveal pervasive effects of culture, learning and language on reasoning and other non-verbal cognitive mechanisms such as categorization and attentional processing (e.g., Pannasch \& Velichkovsky, 2009). More specifically, from a cross-cultural perspective it has been argued that social and cultural differences

ExLing 2018: Proceedings of 9th Tutorial and Research Workshop on Experimental Linguistics, 28-30 August, Paris, Frannce 
strongly affect how people process their environment and more specifically events. Nisbett et al. (2001), as well as Ji and Hohestein (2017) suggest two ways of event processing: one holistic, experiencebased way of thinking associated with the East Asian (i.e. Chinesespeaking) cultures, and one analytic, object-based way of thinking, associated with the Western (i.e. English-speaking) societies.

From a cross-linguistic perspective, similar distinctions have been formulated by Tai (2003): while English-speaking participants have been found more interested in the sequences of a motion event, focusing on processes, agents, objects and action-based constructions, Chinese speakers are more field-focused and their encoding patterns tend to be result-based. This action-based vs. result-based distinction is inspired by the typological distinctions Talmy (2000) formulated in his seminal work on cognitive semantics. According to this work, which goes beyond the East-West distinction, the languages of the world present great differences in the way spatial information is mapped onto lexical and syntactic structures: the so-called Verb-framed languages (e.g. French), allow mostly for lexicalization of the Path component, information about where the event is performed or what is the general result of the displacement without entering into the details often avoiding any specific focal reference to Manner information; the Satellite-framed languages, (e.g. English), mostly allow Path to be expressed in constituents that stand in a sister position to the main verb, lexicalizing Manner of motion, thus focusing on the process and on how motion is actually performed. Additionally, it has been argued that some languages, such as French, allow also some bybrid spatial encodings, with fused Path+Manner verbs, while others, such as Spanish, Chinese, or Greek may present mixed, equipollently-framed or parallel (verb-and satellite-framed) systems of conflation, probably depending on the degree of focality (salience of focal details of the event) within each spatial configuration.

Such differences across and within systems led researchers to address some fundamental questions about the relationship between language/culture and the cognitive mechanisms underlying event representation. Are cognitive processes (e.g., categorization, visual attention etc.) part of an innate independent system or they rather have connections and interact with other systems of processing such as language and culture? And how people, who share the same cultural background (e.g. French, English and Greeks), perceive, categorize and encode motion events? If language only affects language-related experiences, then it is rather unlikely that the building blocks for event 
representation are language-specific. If the cultural experience (western in this case) affects event processing, then no difference should be found across the three language groups. On the other hand, if language impacts language-related behavior but also the representational system, then it is unlikely that solely universal mental categories play a role in event representation. In this case it is expected to find differences even among groups that share the same cultural background with respect to the degrees of focality and sensitivity to details of the displacement.

\section{Method}

60 speakers of three typologically different languages, (English, French, Greek), 20 per language, were tested. They performed three controlled tasks involving motion events: (1) a non-verbal categorization task; (2) a verbal categorization task; and (3) a production task, all coupled with an eye-tracking paradigm for further insights on on-line cognitive processing. In experiment 1 , participants saw a target-video presenting a motion event performed in a certain Manner and along a certain Path (e.g. A woman riding a bicycle into a building). The target was then followed by two video variants: one Manner-congruent ( $A$ woman riding a bicycle out of a building) and one Path-congruent ( $A$ woman riding a scooter into a building). Participants had to choose the variant that looked most like the target as fast as they could by pressing a key. Experiment 2 was exactly the same, except that the target video was replaced by a target sentence. In experiment 3 participants had to describe verbally the events.

The analysis was focused on how participants performed categorization, according to which criterion (Manner or Path) (experiments 1 and 2), what participants expressed, with which linguistic means and in relation to which specific events (experiment 3), and which specific areas of interest (AOI) they were looking at, how many times (numbers of fixations), for how long (visit-durations) and following which visual trajectory (gazepaths).

\section{Results}

The results show that all groups followed the typological patterns of their native language: French participants preferred to lexicalize Path in the utterances leaving Manner either unexpressed or in the periphery; English participants systematically encoded Manner within the main verb and Path in the periphery (with particles or prepositional phrases); while Greek-speaking participants alternated their verb- and satellite-framed constructions (i.e. by using lexicalized Path as well as many peripheral 
devices, preverb configurations, complex Manner-first patterns etc.). French participants were less focal in their non-verbal behaviour than English participants. They made more Path-choices in the categorization tasks, attended more and longer to Path components combining this preference with ballistic (from-source-to-goal) global ways of exploration of the events, as opposed to English who paid less attention to Path and followed a rather focal (linear/step-by-step) strategy for visual processing. Greek participants, depending on the context and the saliency of the components, alternated their visual strategies, showing however that when verbal input is not explicit, overt attention to specific components may differ in fixation counts but not in visit-durations.

\section{Conclusion}

Participants were largely influenced by the typological properties of their native language, not only when performing verbal descriptions but also when making their non-verbal decisions. Despite the fact that they shared the same cultural background (Western), participants categorized and shifted attention mostly based on the features of their language but in some cases, when no verbal input was explicitly involved in the task, the language effect was only superficial. These findings confirm, at least to some extent, the impact of typological constraints in the representational system and support a moderate view about the impact of focality on the Language-Thought relation: some languages (e.g. English) allow for more focal strategies of processing than others (e.g. French), while holistic ways of thinking and ambient processing are not entirely exclusive to Eastern cultures as previously claimed.

\section{References}

Chomsky, N. 2014. The Minimalist Program: 20"th Anniversary edition. Cambridge, MA: MIT Press.

Ji, Y., Hohenstein, J. 2017. Conceptualising voluntary motion events beyond language use. Lingua 195, 57-71.

Kovas, Y., Plomin, R. 2006. Generalist genes. Trends in Cog. Sciences 10(5), 198-203.

Nisbett, R.E., Peng, K., Choi, I., Norenzayan, A. 2001. Culture and systems of thought: holistic vs. analytic cognition. Psychol. Rev. 108, 291-310.

Pannasch, S., Velichkovsky, B.M. 2009. Distractor effect and saccade amplitudes. Visual Cognition 17(6-7), 1109-1131.

Soroli, E., Hickmann, M., Hendriks, H. 2018. Casting an eye on motion events. In Aurnague, M., Stosic D. (Eds.), The semantics of dynamic space in French, 381-438. Amsterdam: John Benjamins.

Tai, J. 2003. Cognitive relativism: Resultative constructions in Chinese. Language and Linguistics 4(2), 301-316.

Talmy, L. 2000. Toward a cognitive semantics. Cambridge, MA: MIT Press. 